\title{
Five-Year Results of a Prospective Multicenter Trial: AdVance XP for Postprostatectomy- Incontinence in Patients with Favorable Prognostic Factors
}

\author{
Jan-Niclas Mumm ${ }^{a}$ Benedikt Klehr ${ }^{a}$ Severin Rodler ${ }^{a} \quad$ Alexander Kretschmer $^{a}$ \\ Theresa Vilsmaier $^{b}$ Thilo Westhofen ${ }^{a}$ Michael Chaloupka ${ }^{a}$ \\ Gerald Bastian Schulz ${ }^{a}$ Christian Gozzic ${ }^{c}$ Peter Rehder ${ }^{d}$ Florian May ${ }^{e}$ \\ Roland Homberg $^{f}$ Christian G. Stief ${ }^{a}$ Markus Grabbertg ${ }^{9}$ Ricarda M. Bauer ${ }^{a}$ \\ ${ }^{a}$ Department of Urology, University of Munich (LMU), Munich, Germany; ${ }^{b}$ Department of Gynecology, University

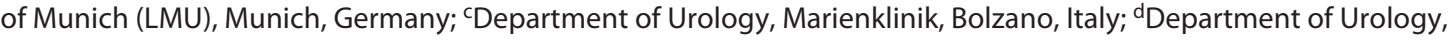

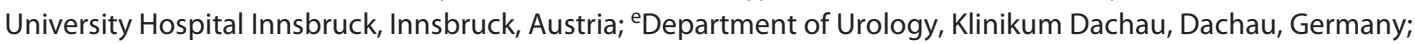 \\ fDepartment of Urology and Paediatric Urology, St. Barbara Hospital Hamm GmbH, Hamm, Germany; ${ }^{9}$ Department \\ of Urology, University Hospital Freiburg, Freiburg, Germany
}

\section{Keywords}

Male stress urinary incontinence $\cdot$ Fixed male sling .

AdVance XP · Patient selection · Postprostatectomyincontinence

\begin{abstract}
Objective: The aim of this study was to assess the security, value, and efficacy of the second-generation AdVance male sling XP (Boston Scientific ${ }^{\circledR}$ ), after implementation in 2010 with advantageous modifications in the sling structure and needle shape, in a prospective multicenter long-term followup study. Methods: In total, 115 patients were included. Exclusion criteria were earlier incontinence (UI) surgery, nocturnal UI, former radiotherapy, or night-time incontinence. We also excluded patients with a functional urethra $<1 \mathrm{~cm}$ in a preoperatively performed repositioning test. A consistent 24-h pad test, International Quality of Life (IQOL) score, visual analog pain scale (VAS), International Consultation-Incontinence Questionnaire (ICIQ-UI SF), International Index of
\end{abstract}

Erectile Function (IIEF-5), International Prostate Symptom Score (IPSS), and Patient Global Impression of Improvement (PGI-I) scores were requested postoperatively. Results: The 24-month follow-up (114 patients) revealed $64.0 \%$ cured and $28.8 \%$ improved patients. Mean urine loss was reduced significantly to $19.0 \mathrm{~g}(p<0.001)$. A mean PGI score of 1.5 and a mean VAS score of 0.2 were obtained. The 60 -month follow-up (59 patients) revealed $57.6 \%$ cured and $25.4 \%$ improved patients. Mean urine loss was reduced significantly to $18.3 \mathrm{~g}(p<0.001)$. A mean PGI score of 1.6 and a mean VAS score of 0.2 were obtained. Conclusions: The AdVance XP displays excellent continence results and secure effectiveness over a 5-year period. Moreover, these data are demonstrating low complication rates and improved quality of life in the long-term use of AdVance XP.

๑ 2021 S. Karger AG, Basel

Ricarda M. Bauer and Markus Grabbert equally contributed to this work. karger@karger.com

(c) 2021 S. Karger AG, Basel

www.karger.com/uin

Karger ${ }^{\prime}=$
Jan-Niclas Mumm

Klinikum der Universität München, Klinik für Urologie

Marchioninistrasse 15

DE-81377 Munich (Germany)

janniclas.mumm@med.uni-muenchen.de 


\section{Introduction}

Postprostatectomy urinary incontinence (PPI) is identified as one of the major risks after radical prostatectomy surgery. A surgical approach of urinary incontinence is recommended when conservative therapy disappoints [1]. The occurrence of PPI being approximately $8.4 \%$ [2], nearly $5 \%$ of the patients after radical prostatectomy even undertake surgical therapy for urinary incontinence within the first 15 years of treatment [3], highlighting the high patient demand in this field. The artificial urinary sphincter (AUS) is an established surgical treatment option for the treatment of PPI [4]; nevertheless, the usage of male slings could in the future supersede its use as the gold standard for certain indications [5].

The nowadays widely used AdVance retrourethral male sling (Boston Scientific ${ }^{\odot}$, MA, USA), firstly presented in 2006 by Rehder and Gozzi [6], was introduced for the management of male stress urinary incontinence (SUI) as a minimally invasive alternative to the AUS. The healing aspect of the repositioning sling is assumed by its functional sustenance of the distal urethral sphincter. This method proved to prevent a continuous passive external compression of the urethra $[7,8]$. Numerous studies in the past have proven the effectiveness and safety of the AdVance sling for treating SUI $[9,10]$. However, the initial production of the AdVance sling system was repeatedly correlated to loss of function in the long-term follow-up period, failing in approximately $20-30 \%$ of cases [11-14] with the need of a subsequent intervention in a total of $12.4 \%$ patients [15]. Analyses so far have predominantly assessed the result and aftermath of the AdVance male sling focusing on the effectiveness and complication profile of the functional male sling in patients with PPI with a maximum follow-up of 48 months in a multicenter study [12, 16-19].

The subsequent generation named AdVance XP male sling launched in 2010 contained numerous changes after large increase in use but associated with inferior success percentages. The novel sling included a restructured mesh with cohesive tensioning fibers in order to improve the material stability. Moreover, small chevron anchors in the sling arms were incorporated, and the system was evolved to enhance fitting of the male pelvic structure by adjusting the tunneling needle shape. Tyvek liners were built in for better strength and to avoid early postoperative slippage $[12,16,20]$.

Given the high patient demand in this field, emphasized by the fact that when given a choice, studies have shown that $92 \%$ of the male patients would choose surgery therapy with a male sling rather than an AUS in order to evade mechanical methods and related manipulation of a scrotal pump [4]; a multicenter long-term study targeting to assess the security and effectiveness regarding the use of the AdVance XP is of high interest. The present analysis is the continuance of a previously described multicenter long-term follow-up study. The objective of this study was to prospectively assess the treatment with the AdVance XP male sling in patients with SUI and the outcome of patients at a prolonged follow-up of 60 months in regard to long-term complications, safety, and efficacy.

\section{Methods}

\section{Patient Acquisition}

Inclusion criteria were patients after radical prostatectomy and persistent SUI. In total, 115 consecutive patients with PPI were included that undertook implantation of an AdVance XP sling between January 2012 and March 2016. The implantation procedures were performed at 6 incontinence centers including Germany, Italy, and Austria. The study was approved after written informed consent by the participating local ethics committees following the ethical standards of the Helsinki Declaration of 1964.

\section{Study Outline}

The implantation of the AdVance XP was accomplished as previously described in our studies $[6,12,19]$. Patients considered were evaluated preoperatively by the operating surgeon and prospectively after $3,6,12,24,36,48$, and 60 months. The preoperative evaluation included, as described before [18]: patient's history, daily pad usage, urodynamics in patients with symptoms of urge incontinence, uroflowmetry, endoscopic evaluation, and sonography for residual urine. For the evaluation preoperatively and at every defined follow-up visit, numerous validated questionnaires were used: International Quality of Life (IQOL) score [21], International Consultation on Incontinence Questionnaire short form (ICIQ-UI SF) [22], International Prostate Symptom Score (IPSS), and International Index of Erectile Function (IIEF-5). Furthermore, the Patient Global Impression of Improvement (PGI-I) [23] and perineal and inguinal postoperative pain based on a visual analog scale (VAS) were measured postoperatively. The ClavienDindo classification (CDC) [24] evaluated and classified complications (Table 3). Furthermore, a standardized questionnaire was sent to all participants. Patients received 1 pad together with a detailed information sheet of how to perform a home 24 -h pad test. The results of the 24 -h pad test together with the daily pad usage were measured in the questionnaires.

"Cure" was defined as 0 pads in $24 \mathrm{~h}$ and a maximum of $5 \mathrm{~g}$ in the 24 -h pad test. "Improvement" was described as a $\geq 50 \%$ reduction in the $24-\mathrm{h}$ pad test. Others outside these ranges were defined as "failures."

\section{Inclusion and Exclusion Criteria}

Inclusion and exclusion criteria for the patient collective have been thoroughly described beforehand [18]. To sum up, patients 
Fig. 1. Postoperative continence status.

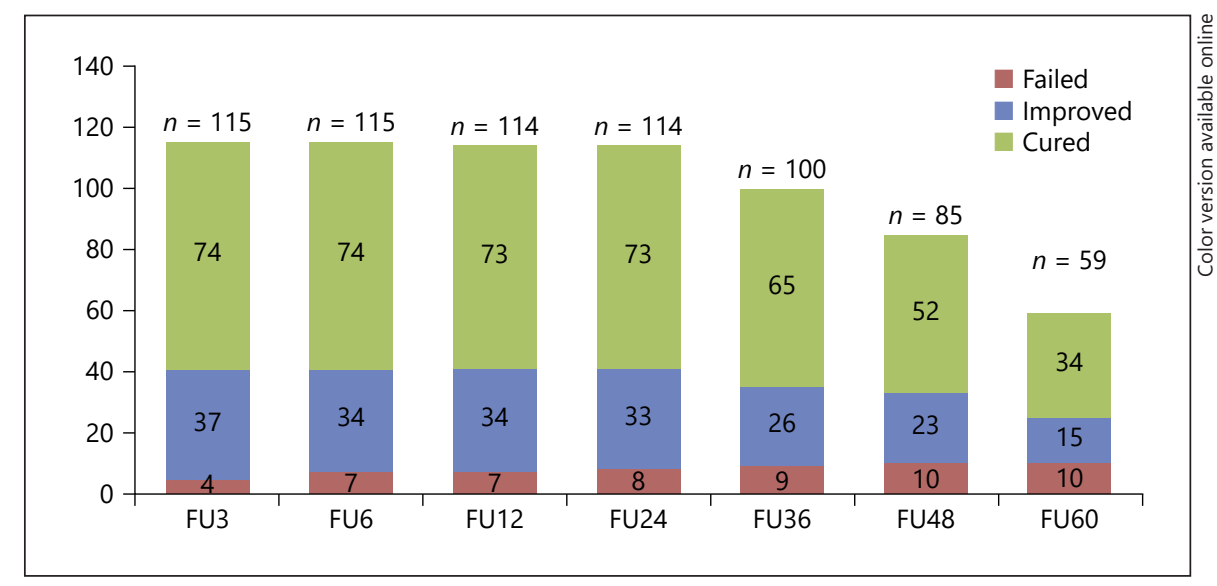

included were defined to have a PPI with a mandatory positive endoscopic evaluation proving a good external sphincter contractility and a minimum coaptive zone of $\geq 1 \mathrm{~cm}$ (positive repositioning test) [25]. The minimum age included was defined at 40 years. As described before, inclusion criteria stated a daily pad usage of up to 8 pads with the definition of a mild-to-moderate incontinence. To continue, the daily pad usage of $1-3$ pads was described as mild incontinence [25]. Nevertheless, there was no limit for urine leakage in the 24-h pad test.

Exclusion criteria included a negative endoscopic evaluation without an adequate coaptive zone, a prior radiation therapy, nocturnal urinary loss (Ul), supine $\mathrm{Ul} \mathrm{III}^{\circ}$, prior incontinence surgery, and previous history of urge Ul. Patients who received other treatment for postoperative incontinence were furthermore excluded.

\section{Statistical Analysis}

Statistical analyses were accomplished using SPSS Statistics 25.0 (IBM Corp., Armonk, NY, USA). The results gathered were recorded and inserted into the SPSS database in the implicit manner. A nonparametric $t$ test (Wilcoxon signed rank test) was used to compare the pre- and postoperative continence status at followup visit. Bivariate correlation (Spearman correlation coefficient) evaluated the influence of patient's variables on the postoperative outcomes. A $p$ value of $<0.05$ indicated statistical significance.

\section{Results}

\section{Patient Cohort}

In total, 115 patients were included that underwent an AdVance XP sling implantation because of SUI after radical prostatectomy from 2012 to 2016. The implantation procedures were performed at 6 incontinence centers including Germany, Italy, and Austria - the 115 patients finally fitting all criteria and considered for this study came from 4 incontinence centers after consideration of exclusion criteria (center $1, n=16$ patients; center $2, n=$ 78 ; center $3, n=14$; and center $4, n=7$ ). There was no
Table 1. Preoperative patient characteristics $(n=115)$

Age, years, median (range)

$69.0(47-82)$

Mode of previous prostate surgery, $n(\%)$

Open radical prostatectomy

$86(75 \%)$

DaVinci/laparoscopic radical prostatectomy $29(25 \%)$

Urine loss in 24-h pad test, g, mean (range)

ICIQ SF, mean (range)

$272.0(42-1,600)$

IQOL, mean (range)

$16.0(8-21)$

$68.0(32-106)$

IIEF-5, mean (range)

IPSS, mean (range)

$5.6(1-25)$

$7.7(0-32)$

BMI, mean (range)

$26(20-38)$

Diabetes, $n$ (\%)

$3 / 115(2.6 \%)$

Neurological diseases, $n(\%)$

BMI, body mass index; ICIQ SF, International Consultation on Incontinence Questionnaire short form; IIEF-5, International Index of Erectile Function; IPSS, International Prostate Symptom Score; IQOL, International Quality of Life.

significant difference in patient outcome in relation to the incontinence center performing the procedure or considering yearly implantation procedures performed. The median age was 69 years (minimum age of 47 and maximum age of 82 years). Twenty-nine months were the median period between radical prostatectomy and the sling implantation (minimum 5 months; maximum 209 months). Table 1 shows baseline patient characteristics. Segments of this table have already been published previously $[12,16,20]$.

\section{Efficacy after Long-Term Follow-Up}

In total, 59 patients (51.3\%) reached 5-year follow-up and were able to be considered for interpretation. Lost to follow-up were 24 patients (20.8\%). Without any relation 
Table 2. Postoperative continence results

\begin{tabular}{lcccc}
\hline & $\begin{array}{l}\text { Baseline } \\
(n=115)\end{array}$ & $\begin{array}{l}24 \text { months } \\
(n=114)\end{array}$ & $\begin{array}{l}48 \text { months } \\
(n=85)\end{array}$ & $\begin{array}{l}60 \text { months } \\
(n=59)\end{array}$ \\
\hline Cured, \% & - & 64.0 & 61.2 & 57.6 \\
Improved, $\%$ & - & 28.9 & 27.1 & 25.4 \\
Failed, \% & - & 7.0 & 11.8 & 16.9 \\
24-h pad test mean, g & 272.0 & $19(\boldsymbol{p}<\mathbf{0 . 0 0 1})$ & $33.8(\boldsymbol{p}<\mathbf{0 . 0 0 1})$ & $18.3(\boldsymbol{p}<\mathbf{0 . 0 0 1 )}$ \\
IPSS, mean & 7.7 & $8.3(p=0.130)$ & $7.8(p=0.254)$ & $8.2(p=0.213)$ \\
IIEF-5, mean & 5.6 & $5.0(p=0.699)$ & $5.2(p=0.445)$ & $4.2(p=0.352)$ \\
ICIQ, mean & 14.9 & $4.8(\boldsymbol{p}<\mathbf{0 . 0 0 1})$ & $5.3(\boldsymbol{p}<\mathbf{0 . 0 0 1})$ & $5.0(\boldsymbol{p}<\mathbf{0 . 0 0 1})$ \\
IQOL, mean & 67.3 & $97.0(\boldsymbol{p}<\mathbf{0 . 0 0 1})$ & $96.9(\boldsymbol{p}<\mathbf{0 . 0 0 1})$ & $96.8(\boldsymbol{p}<\mathbf{0 . 0 0 1})$ \\
PGI, mean & - & 1.5 & 1.6 & 1.6 \\
VAS, mean & - & 0.2 & 0.2 & 0.2 \\
\hline
\end{tabular}

Bold type denotes significance. ICIQ, International Consultation on Incontinence Questionnaire; IIEF-5, International Index of Erectile Function; IPSS, International Prostate Symptom Score; IQOL, International Quality of Life; PGI, Patient Global Impression of Improvement; VAS, visual analog scale.

to sling implantation, 2 participants of this study (1.7\%) deceased throughout the follow-up time frame. For the sake of completeness, our previous studies have already reported on the outcomes of the same patient cohort after set follow-up periods; in our previous studies, $115 \mathrm{pa}$ tients were included after 3-month follow-up, 115 also after 6-month follow-up, 114 participants of the same patient cohort were included in the 12-month and also the 24-month follow-up, 100 patients were followed up after 36 months, 85 patients could be included after 48 months, and 59 patients after the 60 -month follow-up period. This study focuses on the follow-up of the same patient cohort after a 5-year follow-up period. No further patients have been added.

After a long-term follow-up of 5 years, $57.6 \%$ of the patients were classified as cured and $25.4 \%$ of the patient collective statistically improved. Furthermore, categorized as unsuccessful were $16.9 \%$ of the participants. The outcomes for each follow-up time period are visualized in Figure 1, demonstrating the postoperative continence status. Mean PGI was 1.5 after 3-month follow-up, 1.5 after 2-year follow-up, 1.6 after 4 years, and 1.6 after the 5 -year period.

In terms of functional outcome, the 5-year follow-up data displayed statistically significant results. The urine loss was significantly decreased in the 24 -h pad test. The ICIQ-UI SF survey also revealed a decline regarding the urinary incontinence grade at every time point of the follow-up period (see Table 2) exclusive of statistical alterations in regard to the 24-h pad test throughout the testing time points ( $p>0.005$, each). The quality of life measured by the IQOL test improved significantly. No statistically significant changes in erectile function (IIEF-5) or increased irritative symptoms assessed by the IPSS occurred as shown in Table 2 .

In the subgroup evaluation, there were no statistically significant findings ( $p>0.05$, each). Subgroups included the influence of age, the category of radical prostatectomy procedure such as the implanting physician itself, the body mass index, the preoperative IPSS, and the preoperative ICIQ-UI SF results. Further subcategories that showed no statistically significant results were the preoperative urine loss tested by the 24 -h pad test to be continued by pad usage on postoperative IQOL and continence status at maximum follow-up.

Improvement and cure proportions did not vary significantly over each follow-up period. The continence status seemingly does not seem to worsen over time ( $p>$ 0.005 , each).

\section{Complications}

No intraoperative complications occurred. 0.5 was the mean pain VAS score after the 3-month follow-up and 0.2 after the 60-month evaluation. No participant was in need of pain relief therapy in the prolonged postoperative phase for over 4 weeks. Furthermore, neither severe nor explicit postoperative complications were described (in regard to $\mathrm{CDC}$ grade IV-V [26]). No consecutive infections or erosions in the follow-up period were reported that potentially needed or ended in the explantation of the sling.

As previously reported, a total of 7 device-related complications occurred, nevertheless, not in the time period 
Table 3. Device-related postoperative complications defined by CDC

\begin{tabular}{llllllll}
\hline CDC (grade) & I & IIa & IIb & IIIa & IIIb & VI & V \\
\hline Total $(n=115)$ & 0 & 5 & 1 & 0 & 9 & 0 & 0 \\
Percentage & - & 4.3 & 0.9 & - & 7.8 & - & -
\end{tabular}

CDC, Clavien-Dindo classification.

of the extended follow-up to 60 months. Newly described urge was recorded throughout the follow-up period in the first year after sling implantation that required medical treatment by a total of 5 study participants ( $4.3 \%$ complication grade IIa in respect to the CDC). One patient $(0.9 \%)$ was treated with UTI postoperatively (grade IIb in regard to $\mathrm{CDC}$ ). During the investigated time frame, dissection of 1 sling arm due to urinary retaining or urge complications related to subvesical obstruction by overtensioning of the sling was implemented in 9 participants (7.8\%; grade IIIb with respect to CDC). One patient received implantation of an AUS due to worsening of urinary incontinence symptoms subsequently to sling implantation (0.9\%; grade III in regard to CDC). Table 3 visualizes the above-described findings.

Radiation therapy was conducted in a total of 3 patients $(2.6 \%)$ in the follow-up period due to pathogen relapse of the underlying disease. Because of radiation, 2 of those patients reported the return of urinary incontinence symptoms. In the previous analysis describing the prolonged follow-up of 60 months, no further late onset complications were reported.

\section{Discussion}

The AdVance XP was firstly launched in 2010, providing a dynamic sustenance to the sphincter during pressure by increasing the coaptive zone in the sphincteric part of the urethra. Device changes intending to facilitate implantation and improving postoperative stability have been implemented [7]. Its use has emerged to a well-established therapeutic tool in the management of PPI. For the treatment of PPI, the AdVance sling was firstly introduced in 2006. To this point, there are 2 studies in place comparing the effectiveness of the AdVance in contrast to the AdVance XP sling. Until now, the studies did not prove significant progressive effectiveness in favor of the AdVance XP sling. Nevertheless, it emphasized advan-

AdVance $\mathrm{XP}^{\circledast}$ for Postprostatectomy-

Incontinence tages, for example, in the decrease of failure frequency in overweight participants $[27,28]$. A study comparing patients treated with either the AdVance sling or the AdVance XP sling revealed a total cure rate of $77 \%$ following a median follow-up of 49 months [20, 29]. From a patient's point of view, the AdVance XP sling shows undeniable advantages such as no mechanical components and the ability to function without relying on patient-related cognition [14].

Firstly introduced in 1972, the AUS is to this point considered as the gold standard for treating PPI [15]. However, in a single-center patient cohort containing 218 patients treated with AUS, 27\% needed surgical revision or even explantation within the first 5 years [30], highlighting the fact that the AUS does not come without disadvantages and complications. Studies have disclosed the deficiency of verification for the surgical management of PPI, being particularly unsatisfying for male sling systems, while patients demand remains high [12].

To this day, there are only a few equivalent studies specifying adequate data of midterm to long-term followup of the AdVance XP male sling in relation to treatment of PPI. Our study cohort provides the longest follow-up of 60 months in a multicenter study cohort after AdVance XP implantation, allowing new insights into long-term durability, efficacy, and safety.

Rehder et al. [33] described a long-term follow-up of the AdVance sling after 36 months, showing cure rates of $53.0 \%$ in total, including a cured and improved rate of $76.8 \%$ (patients defined as cured using $0-1$ pads/24 h). Zemp et al. [14] were able to show continence rates of $82.3 \%$ in patients with no identified risk factors for AdVance sling failure, described in a mean follow-up of 43 months. Consistent with the findings of Zuckerman et al. [31] stating a cure rate of $62 \%$ of patients either described as cured or improved after a median follow-up of 36 months, their findings indicated declining functional rates over the described follow-up time [11]. These findings nevertheless concentrated on the significant cure rates after the implantation of the AdVance male sling. The described studies have led to the introduction of the modified transobturator-retroluminal-repositioning sling structure, also known as AdVance XP.

In regard to these findings, our study for the first time allows a well-defined indication that the second-generation sling demonstrates constant continence outcomes over a prolonged follow-up time. Bauer et al. [12, 32] emphasized a cure rate of $69.3 \%$ after 24 months and $66 \%$ after 36 months. The follow-up after 48 months determined a $71.7 \%$ cure rate [19]. The present study after a 60 -month 
follow-up period is furthermore coherent with the previously described outcomes for the AdVance XP, stating a cure rate of $57.6 \%$ and the exact description of continence in our current study of 0 pads $/ 24 \mathrm{~h}$ together with a maximum of 5-g urine loss in the 24-h pad test. A significant outcome of the present analysis is that the efficiency and duration after AdVance XP implantation does not seem to statistically reduce in the first 5 years. Furthermore, these findings are stable with the outcomes of our earlier reports considering long-term outcomes after AdVance implantation [19]. These findings reveal the efficacy and stability of the AdVance XP in the therapy of PPI.

We continued to only include and follow-up patients out of our highly selected patient cohort with very strictly applied selection criteria in contrast to most studies described before. The definition of continence was stated beforehand: cured patients defined with 0 pads $/ 24 \mathrm{~h}$ together with a 24 -h pad test showing $5 \mathrm{~g}$ of maximum urine loss. Most analyses so far evaluated the efficacy of the AdVance XP male sling on individually chosen patients, for instance, exclusive of any previous incontinence surgeries or prior pelvic radiation therapy $[25,33,34]$. In contrast, the present study once again highlights that in our patient cohort, no association to failure to achieve continence was made in regard to the age, preoperative quantity of urinary incontinence, or BMI. Consequently, a cutoff urine loss cannot be stated to predict a favorable result [19].

The postoperative complications rates were low, and no further patient suffered from de novo urge after the postoperative period $[16,32,35,36]$. To continue, no statistical substantial difference in regard to the IPSS was shown. Further complications did not follow in the longterm follow-up, supporting the previously described results for the AdVance and the AdVance XP [12, 16, 19, $20,32]$. No erosions or explantations of neither a whole sling system nor fragments of the sling were described throughout the follow-up period. Also, no participant stated extensive discomfort. No statistically significant postoperative deviations in erectile function were measured with the IIEF- 5 score. The patient satisfaction was furthermore stable throughout the follow-up period proven by the PGI and IQOL scores. Merely, 53\% of the men questioned suffering of incontinence after radical prostatectomy would again choose the surgical treatment option when asked at 5 years or more after treatment. This proves the satisfaction of patients is an important feature and goal in the future in order to enable a longterm quality of life to prostate cancer survivors.

Limitation of the study, carried out as a single-arm study, is the missing of a control group. Furthermore, at the moment, the 60-month follow-up was only reached by $59 / 115$ patients, making it $67.8 \%$ of the total patient cohort treated. The highly selected patient selection at the start point of this study demarks a further limitation as described in our previous studies or in contrast highlights the results in well-selected patients especially regarding the still open question "what is the ideal patient?"

\section{Conclusion}

The Advance XP displays excellent continence results and secure effectiveness over a 5 -year period. Moreover, these data are demonstrating low complication rates and improved quality of life in the long-term use of AdVance XP.

\section{Statement of Ethics}

We received written informed consent from all patients included, formerly to this study (Reference No. 522-11). All local ethics boards contributing to this study permitted the prospective multicenter long-term follow-up analysis. The ethical principles set and its amendments in the 1964 Declaration of Helsinki have all been met.

\section{Conflict of Interest Statement}

Ricarda M. Bauer proclaims lectures, consultancy effort, and the contribution in clinical trials for AMS/Boston Scientific. P. Rehder and C. Gozzi are the coinventors of the technique. Nevertheless, they do not claim intellectual property. P. Rehder and C. Gozzi also declare lectures, consultancy employment, and involvement in clinical trials for AMS. R. Homberg declares consultancy work such as lectures for AMS. There are no conflicts of interest stated by all other authors.

\section{Funding Sources}

The authors did not receive any funding.

\section{Author Contributions}

Study concept and design: R.B., C.S., and C.G.; acquisition of data: J.M., R.B., F.M., M.G., and A.K.; analysis and interpretation of data: J.M., M.G., R.B., C.G., and C.S.; drafting of the manuscript: J.M.; critical revision of the manuscript for important intellectual content: S.R., T.V., T.W., and G.S.; statistical analysis: J.M., B.K., and M.C.; obtaining funding: none; administrative, technical, or material support: F.M. and R.H.; and supervision: R.B, C.S., and C.G. 


\section{References}

1 Lucas MG, Bosch RJ, Burkhard FC, Cruz F, Madden TB, Nambiar AK, et al. EAU guidelines on surgical treatment of urinary incontinence. Actas Urol Esp. 2013;37(8): 459-72.

2 Stanford JL, Feng Z, Hamilton AS, Gilliland FD, Stephenson RA, Eley JW, et al. Urinary and sexual function after radical prostatectomy for clinically localized prostate cancer: the Prostate Cancer Outcomes Study. JAMA. 2000;283(3):354-60.

3 Nam RK, Herschorn S, Loblaw DA, Liu Y, Klotz LH, Carr LK, et al. Population based study of long-term rates of surgery for urinary incontinence after radical prostatectomy for prostate cancer. J Urol. 2012;188(2):502-6.

4 Kumar A, Litt ER, Ballert KN, Nitti VW. Artificial urinary sphincter versus male sling for post-prostatectomy incontinence: What do patients choose? J Urol. 2009;181(3):1231-5.

5 Hoy NY, Rourke KF. Stemming the tide of mild to moderate post-prostatectomy incontinence: a retrospective comparison of transobturator male slings and the artificial urinary sphincter. Can Urol Assoc J. 2014;8(78):273-7.

6 Rehder P, Gozzi C. Transobturator sling suspension for male urinary incontinence including post-radical prostatectomy. Eur Urol. 2007;52(3):860-6.

7 Rehder P, Staudacher NM, Schachtner J, Berger ME, Schillfahrt F, Hauser V, et al. Hypothesis that urethral bulb (corpus spongiosum) plays an active role in male urinary continence. Adv Urol. 2016;2016:6054730.

8 Kretschmer A, Hübner W, Sandhu JS, Bauer RM. Evaluation and management of postprostatectomy incontinence: a systematic review of current literature. Eur Urol Focus. 2016;2(3):245-59.

9 Bauer RM, Mayer ME, May F, Gratzke C, Buchner A, Soljanik I, et al. Complications of the AdVance transobturator male sling in the treatment of male stress urinary incontinence. Urology. 2010;75(6):1494-8.

10 Bauer RM, Mayer ME, Gratzke C, Soljanik I, Buchner A, Bastian PJ, et al. Prospective evaluation of the functional sling suspension for male postprostatectomy stress urinary incontinence: results after 1 year. Eur Urol. 2009; 56(6):928-33

11 Papachristos A, Mann S, Talbot K, Moon D. AdVance male urethral sling: medium-term results in an Australian cohort. ANZ J Surg. 2018;88(3):E178-82.

12 Bauer RM, Grabbert MT, Klehr B, Gebhartl B, Gozzi C, Homberg R, et al. 36-month data for the AdVance $\mathrm{XP}^{\oplus}$ male sling: results of a prospective multicentre study. BJU Int. 2017; 119(4):626-30

13 Cornu JN, Sèbe P, Ciofu C, Peyrat L, Cussenot O, Haab F. Mid-term evaluation of the transobturator male sling for post-prostatectomy incontinence: focus on prognostic factors. BJU Int. 2011;108(2):236-40.

14 Zemp L, Tong S, Hoy N, Rourke KF. Preoperative pad usage is independently associated with failure of non-adjustable male trans-obturator slings in otherwise well-selected patients. Can Urol Assoc J. 2019;13(4):120-4.

15 Ye H, Haab F, de Ridder D, Chauveau P, Becker A, Arano P, et al. Effectiveness and complications of the AMS AdVance male sling system for the treatment of stress urinary incontinence: a prospective multicenter study. Urology. 2018;120:197-204.

16 Kretschmer A, Grabbert M, Sommer A, Stief CG, Bauer RM. Mid-term outcomes after AdVanceXP male sling implantation. BJU Int. 2016;118(3):458.

17 Bauer RM, Rutkowski M, Kretschmer A, Casuscelli J, Stief CG, Huebner W. Efficacy and complications of the adjustable sling system ArgusT for male incontinence: results of a prospective 2-center study. Urology. 2015; 85(2):316-20.

18 Bauer RM, Gozzi C, Klehr B, Kretschmer A, Grabbert M, Rehder P, et al. AdVanceXP male sling: 2-year results of a multicentre study. World J Urol. 2016;34(7):1025-30.

19 Grabbert M, Mumm JN, Klehr B, Kretschmer A, Gebhartl P, Gozzi C, et al. Extended followup of the AdVance XP male sling in the treatment of male urinary stress incontinence after 48 months: results of a prospective and multicenter study. Neurourol Urodyn. 2019 Sep; 38(7):1973-8

20 Bauer RM, Kretschmer A, Stief CG, Füllhase C. AdVance and AdVance XP slings for the treatment of post-prostatectomy incontinence. World J Urol. 2015;33(1):145-50.

21 Bushnell DM, Martin ML, Summers KH, Svihra J, Lionis C, Patrick DL. Quality of life of women with urinary incontinence: crosscultural performance of 15 language versions of the I-QOL. Qual Life Res. 2005;14(8):190113.

22 Avery K, Donovan J, Peters TJ, Shaw C, Gotoh M, Abrams P. ICIQ: a brief and robust measure for evaluating the symptoms and impact of urinary incontinence. Neurourol Urodyn. 2004;23(4):322-30.

23 Twiss CO, Fischer MC, Nitti VW. Comparison between reduction in 24-hour pad weight, International Consultation on IncontinenceShort Form (ICIQ-SF) score, International Prostate Symptom Score (IPSS), and PostOperative Patient Global Impression of Improvement (PGI-I) score in patient evaluation after male perineal sling. Neurourol Urodyn. 2007;26(1):8-13

24 Clavien PA, Barkun J, de Oliveira ML, Vauthey JN, Dindo D, Schulick RD, et al. The Clavien-Dindo classification of surgical complications: five-year experience. Ann Surg. 2009; 250(2):187-96.
25 Bauer RM, Gozzi C, Roosen A, Khoder W, Trottmann M, Waidelich R, et al. Impact of the 'repositioning test' on postoperative outcome of retroluminar transobturator male sling implantation. Urol Int. 2013;90(3):3348.

26 Dindo D, Demartines N, Clavien PA. Classification of surgical complications: a new proposal with evaluation in a cohort of $6336 \mathrm{pa}-$ tients and results of a survey. Ann Surg. 2004 240(2):205-13.

27 Cornu JN, Batista Da Costa J, Henry N, Peyrat L, Beley S, Haab F. Comparative study of AdVance and AdVanceXP male slings in a tertiary reference center. Eur Urol. 2014;65(2): 502-4.

28 Soljanik I, Bauer RM, Stief CG, Gozzi C, Becker AJ. Pelvic floor muscle function is an independent predictor of outcome after retrourethral transobturator male sling procedure. World J Urol. 2015;33(8):1143-9.

29 Husch T, Kretschmer A, Thomsen F, Kronlachner D, Kurosch M, Obaje A, et al. The AdVance and AdVanceXP male sling in urinary incontinence: Is there a difference? World J Urol. 2018;36(10):1657-62.

30 Lai HH, Hsu EI, Teh BS, Butler EB, Boone TB. 13 years of experience with artificial urinary sphincter implantation at Baylor College of Medicine. J Urol. 2007;177(3):1021-5.

31 Zuckerman JM, Edwards B, Henderson K, Beydoun HA, McCammon KA. Extended outcomes in the treatment of male stress urinary incontinence with a transobturator sling. Urology. 2014;83(4):939-45.

32 Bauer RM, Gozzi C, Klehr B, Kretschmer A, Grabbert M, Rehder P, et al. AdVance XP male sling: 2-year results of a multicentre study. World J Urol. 2016 Jul;34(7):1025-30.

33 Rehder P, Haab F, Cornu JN, Gozzi C, Bauer RM. Treatment of postprostatectomy male urinary incontinence with the transobturator retroluminal repositioning sling suspension: 3-year follow-up. Eur Urol. 2012;62(1):1405.

34 Sturm RM, Guralnick ML, Stone AR, Bales GT, Dangle PP, O'Connor RC. Comparison of clinical outcomes between "ideal" and "nonideal" transobturator male sling patients for treatment of postprostatectomy incontinence. Urology. 2014;83(5):1186-8.

35 Kowalik CG, DeLong JM, Mourtzinos AP. The advance transobturator male sling for post-prostatectomy incontinence: subjective and objective outcomes with 3 years follow up. Neurourol Urodyn. 2015;34(3):251-4.

36 Collado A, Domínguez-Escrig J, Ortiz Rodríguez IM, Ramirez-Backhaus M, Rodríguez Torreblanca C, Rubio-Briones J, et al. Functional follow-up after Advance ${ }^{\circ}$ and Advance $\mathrm{XP}^{\star}$ male sling surgery: assessment of predictive factors. World J Urol. 2019;37(1):195200
AdVance $\mathrm{XP}^{\circledR}$ for PostprostatectomyIncontinence
Urol Int 2021;105:421-427

DOI: $10.1159 / 000512881$ 